\title{
BATERÍA LURIA INICIAL Y DESARROLLO DE LAS FUNCIONES PSICOLÓGICAS SUPERIORES INITIAL BATTERY LURIA AND DEVELOPMENT OF HIGHER PSYCHOLOGICAL FUNCTIONS
}

\author{
YASER RAMÍREZ BENÍTEZ
}

Cómo referenciar este artículo/How to reference this article:

Ramírez Benítez, Y. (2014). Batería Luria Inicial y desarrollo de las funciones psicológicas superiores [Initial Battery Luria and development of Higher Psychological Functions]. Acción Psicológica, 11(1), 69-78. http://dx.doi.org/10.5944/ap.1.1.13868

\section{Resumen}

La batería neuropsicológica Luria Inicial es un instrumento válido para evaluar y seguir el desarrollo de las funciones psicológicas superiores en la edad preescolar. El modelo neuropsicológico de Luria ofrece la posibilidad para conocer las funciones superiores del niño. Metodología: La investigación pretende determinar la utilidad clínica del instrumento Luria Inicial en la población cubana preescolar con alteraciones del neurodesarrollo. El objetivo se cumplió con la revisión de los trabajos científicos publicados en la fecha 2006-2013 en bases de datos electrónica (Scielo, EBSCO host, LILACS, Medigraphic). Resultados: El instrumento tiene una efectiva utilidad clínica para conocer el perfil neuropsicológico del niño preescolar con alteraciones del neurodesarrollo. Los resultados en varias poblaciones (TDAH, bajo peso al nacer, desórdenes metabólicos) informan que el acto motor con intencionalidad, la inhibición conductual y la organización espacial son habilidades diana a cualquier evento que interrumpan el neurodesarrollo. La adquisición de la función reguladora del habla predice las condiciones preacadémicas del niño en conjunto con el estado real de las funciones superiores. La utilidad clínica del instrumento está en la posibilidad de describir-explicar el estado real de las funciones superiores y su potencial: el niño puede ejecutar e imitar movimientos, dibujar, reconocer los colores y hablar (estado real), pero si no organiza esas ejecuciones a través de la función reguladora del habla (estado potencial) estará sujeto a errores y a no aprender por orientaciones del adulto en la etapa escolar. La línea de pensamiento que ofrece Luria para estudiar a los niños es válida y confiable para diagnosticar y rehabilitar las secuelas del sistema nervioso en desarrollo.

Palabras clave: Luria Inicial; neuropsicología infantil; neurodesarrollo; modelo neuropsicológico de Luria; preescolar.

Correspondencia: Profesor Universidad de Cienfuegos «Carlos R Rodríguez». Investigador del Servicio de Neuropsicología. Centro Docente de Rehabilitación del Neurodesarrollo, Cárdenas, Matanzas, Cuba. Email: yaser@deleg.perla.inf.cu 


\begin{abstract}
The neuropsychological battery Initial Luria is a valid instrument to evaluate and follow the superior neurological function development at preschooler age. Luria's neuropsychological model offers the theory and practical possibilities of knowing infant's potential and real superior functions. Methodology: The investigation purport determines clinical use for Luria Initial instrument in neurodevelopment alterations cuban children. The revision original article from 2006-2013 in electronic data base (Scielo, EBSCO host, LILACS, Medigraphic). This instrument has affective clinical utility in Cuba, scenically in population neurodevelopment alterations. The results several populations (TDAH, low birth weight, metabolic disorders) inform that the motor act intentionality, conduct inhibition and spacial organization are target skills in every event that interrupt the neurodevelopment. Acquirement of speak regulation function predicts preacademic infant conditions together the real state of superior functions. This instrument clinical utility is in the possibility of describing - explaining the real superior function real state and its potential: the infant can move and copy movement, draw, recognize colors and speak (real functions age state), but if the infant does not organize those actions trough regulator oral language (potential functions state), at will make mistakes and not learning through adult orientations at scholar age. The theory Luria offers study this nervous system development.
\end{abstract}

Keyword: Initial Luria; children neuropsychology; neurodevelopment; Luria neuropsychological model; preschool.

\section{Introducción}

El instrumento neuropsicológico Luria Inicial surge de la experiencia acumulada por otras investigaciones en edades infantiles (Christensen, 1987; Korkman, 1988; Lezak, 1984; Luria, 1982; Portellano, Mateos, Martínez, Granados y Tapia, 2000).
Los autores del instrumento parten de la experiencia acumulada y de la propia para organizar un grupo de subpruebas sensibles a las alteraciones del neurodesarrollo en la etapa preescolar. La experiencia propia surge de la investigación con el instrumento Luria DNI (Diagnóstico Neuropsicológico Infantil) y el aumento de poblaciones infantiles con alteraciones del sistema nervioso (Inmadurez/Disfunción).

Los objetivos de los autores fue, en primer lugar, crear un instrumento que fuera extensión del modelo neuropsicológico de Luria para edades inferiores y ajustada a los avances evolutivos de la etapa (Motricidad, Lenguaje oral, Rapidez de procesamiento, AprendizajeMemoria y Lateralización manual). En segundo lugar se destaca la importancia de la maduración cerebral que las edades preescolares alcanzan en cuanto a bases neurales del desarrollo perceptivo - motor y cognitivos y en tercer lugar se intenta que las escalas identifiquen aptitudes y habilidades preacadémicas. (Manga y Ramos, 2006).

La comunidad científica aceptó la propuesta de los doctores con varios trabajos científicos que resaltan la utilidad práctica del instrumento (Bausela, 2008, 2009a, 2009b; Ramírez, 2008; Ramírez y González, 2008; Ramírez y Marchena, 2008, 2009; Ramírez y Novoa, 2008; Ramírez y Díaz, 2013; Ramírez, Díaz, Hernando y Samoano, 2011; Ramírez, Díaz, Ramos y Manga, 2013) y la visión teórica del modelo neuropsicológico en edades preescolares.

Los estudios neuropsicológicos realizados en Cuba se justifican por la baja tasa de mortalidad que se ha experimentado en los últimos 10 años. El aumento de poblaciones con riesgo biológico/cognitivo invita a los profesionales a construir perfiles neurocognitivos con fines de garantizar el éxito escolar.

La madurez neuropsicológica ha sido la principal variable que buscan los investigadores para relacionarla con el futuro desempeño escolar (C. Campo, Tuesca y L. Campo, 2012, Guerrero, Guillén y Sato, 2011; Portellano et al., 2000; Raz, Newman, Dabastos, Peters y Batton, 2014), aunque por otra parte el control de las ejecuciones con símbolos puede ser ele- 
mental para organizar la conducta y el currículo escolar del niño preescolar. (Aguilera, Ostrosky y Camarena, 2012; Guaita, 2012; Solovieva, Lázaro y Quintanar, 2012; Solovieva y Quintanar, 2013).

Considerando la importancia de la madurez alcanzada por el niño preescolar para avanzar en la próxima etapa, la investigación pretender realizar una revisión teórica sobre la utilidad clínica del instrumento neuropsicológico Luria Inicial en la población cubana preescolar con riesgo biológico (rasgos TDAH, desordenes metabólicos y bajo peso al nacer).

\section{Metodología}

La investigación utilizó como unidad de análisis 8 artículos originales publicados en diferentes revistas.

El procedimiento se llevó a cabo mediante una recopilación de información en las bases de datos electrónicas (Scielo, EBSCO host, LILACS, Medigraphic). El año de publicación de los artículos debía estar comprendido entre el 2006-2013 y el idioma de publicación ingles/ castellano. La búsqueda se realizó en los meses de enero y abril de 2013.

Se analizaron los datos de acuerdo al objetivo de la revisión: artículos originales donde se utilizó el instrumento neuropsicológico Luria Inicial. Se excluyeron los trabajos publicados en población no cubanas.

\section{Resultados}

Las investigaciones realizadas en Cuba muestran no solo la validez del instrumento para ser aplicado en poblaciones con documentada alteración del neurodesarrollo (Ramírez y Díaz, 2013), también su utilidad para identificar puntos débiles en poblaciones que no está documentado el daño cerebral: aquellos niños que su déficit tiene una naturaleza silente por errores madurativos o por baja estimulación educativa y aparece en años posteriores a la causa real. (Ramírez y Díaz, 2013).
Los estudios reportaron que el desarrollo de las habilidades viso espaciales, la inhibición conductual y el acto motor con intenciones voluntarias son habilidades superiores «dianas» a cualquier evento que interrumpa el neurodesarrollo (Ramírez y Díaz, 2013). Resultado que muestra la importancia que tienen la maduración de los lóbulos frontales/parietales en la etapa y en las adquisiciones que deben establecerse en la edad.

El resultado es coherente con otros estudios que reportan la susceptibilidad de las funciones motoras y la inhibición en la etapa (Campo 2010; González y Ostrosky, 2012; Lozano y Ostrosky, 2012; Quintanar, Gómez, Solovieva y Bonilla, 2011; Sandoval y Ostrosky, 2012; Solovieva, Lázaro y Quintanar, 2008). Ambas son factores que responden a las funciones ejecutivas en desarrollo y van ganando complejidad en la medida que el niño asuma el control de las acciones desde los 4 años de edad con el lenguaje oral.

\section{Bajo peso al nacer}

El niño bajo peso $1.500-2.500 \mathrm{~g}$ en la etapa preescolar es una población con pocos estudios publicados, quizás por ser el grupo más cercano a la norma de los tres grupos (menor a $1000 \mathrm{~g} ; 1.000-1.500 \mathrm{~g}$ y $1.500-2.500 \mathrm{~g}$ ). La publicación encontrada sobre el bajo peso 1.5002.500 g reportó la existencia de signo negativo en el desarrollo de las regiones parietales (Ramírez, 2013). Los antecedentes en las historias clínicas informan que la población comienza con ligeros resultados por debajo de 100 en el coeficiente de desarrollo (Prueba Brunet Lezine), para luego continuar (edad preescolar) con bajos puntuaciones en tareas que demandan el factor visoespacial (prueba Bender y Luria Inicial). El acto motor pierde sus principales coordenadas para ejecutar con precisión sus acciones.

El perfil cognitivo que muestra el niño $1.500-2.500 \mathrm{~g}$ es menos discapacitante que las demás poblaciones por debajo a $1.500 \mathrm{~g}$ (Bjuland, Løhaugen, Martinussen y Skranes, 2012; Fernandes et al., 2012; Kwinta et al., 2012; 
Walhovd et al., 2012), quizás por la condición clínica «bajo peso al nacer» y el efecto de la prematuridad.

El factor espacial puede ser una condición negativa para comenzar el aprendizaje académico en esta población: seguir la lectura, hacer los trazos de las letras y los números, escribir en el reglón exigido y mantener la atención aún cuando aparezca la fatiga.

En la medida que la condición bajo peso al nacer sea más grave las alteraciones son más evidente. De manera general, la literatura reporta que el perfil neurocognitivo del niño bajo peso se caracteriza por bajo desempeño en tareas intelectuales (Hutchinson et al., 2013; Takayanagi et al., 2013), académicas (Adair et al., 2013; Andrews et al., 2009; Hutchinson et al., 2013) y discapacidades ejecutiva (Griffiths et al., 2013, Ritter, Perrig, Steinlin y Everts, 2013), aunque también muestran bajo control/ regulación emocional (Drvaric et al., 2013; Lee y Houk, 2012).

La población puede recibir tratamiento temprano y tener resultados positivos sobre su neurodesarrollo (N. Li, Kang et al., 2013) y son sensibles a modificar su rendimiento cognitivo ante entrenamiento desde la edad preescolar (Lohaugen, Austeng, Brubakk y Skranes, 2013).

\section{TDAH}

Otra población que abunda en la consulta son los niños con rasgos TDAH (trastorno de atención con hiperactividad) en la edad preescolar. El DSM -IV informa que se debe diagnosticar el TDAH en niños después de los 6 años, aunque en la clínica se conoce que hay signos que potencialmente delatan un futuro TDAH.

Estudios actuales revelan que los rasgos de TDAH son evidentes en la etapa preescolar -déficit de atención, psicomotores, viso espacial- (Cherkasova et al., 2013; Goodman y Livingston, 2013; Pauli-Pott et al., 2013; Schmiedeler y Schneider, 2013) y pueden influir en el futuro desempeño escolar. (Charach et al., 2013; Riddle et al., 2013; Serrano-Troncoso et al., 2013).
El instrumento Luria Inicial permitió confeccionar un perfil de puntos débiles: $100 \%$ con dificultades en la inhibición conductual -test 4y en las habilidades viso espaciales -test 2 y 5 y un $60 \%$ en el desarrollo del acto motor voluntario -test 1, 2 y 3-(Ramírez et al., 2011).

El análisis cualitativo de los subtest que conforman el instrumento Luria Inicial reveló que no hay un subtest específico que evalué la atención. Un estudio realizado en niños con rasgos TDAH informó que hay subtest del dominio ejecutivo que correlaciona con pruebas específicas de atención sostenida: el subtest motricidad manual -test 1- correlacionó con la atención sostenida auditiva -Beta. 0.81- y con la atención selectiva (prueba de interferencia tipo Stroop) -Beta. 0.81-, el subtest organización espacial -test 5- correlacionó con la atención sostenida visual -Beta. 0.95- (Ramírez et al., 2011).

La investigación concluyó que comprender órdenes orales para organizar y ejecutar el acto motor -test 1-, adquirir la inhibición conductual para «frenar» conductas motoras a través del lenguaje oral -test 4- y organizar el acto motor a través de coordenadas espaciales -test 5- son habilidades superiores que demandan abundantes recursos atencionales para su adecuado funcionamiento, por lo cual las pruebas que la evalúan son fuertes para conocer el estado de los procesos atencionales y ejecutivos en la edad.

El resultado pone en evidencia lo planteado por Christensen cuando comenta los movimientos con las manos son un buen ejemplo para conocer la capacidad de programar y regular la actividad propositiva del niño. Los procesos atencionales en el niño preescolar deben estudiarse integrados a los avances ejecutivos de la etapa, pues permitirán vigilar y regular acciones con un fin.

\section{Desórdenes metabólicos}

En iguales condiciones, el instrumento tuvo una aceptación en los servicios de Endocrinología del Hospital Pediátrico de Cienfuegos en los años 2007-2009. La alta prevalencia de desórdenes metabólicos congénitos en la so- 
ciedad es un reto científico potencial, lo cual exige un servicio de neuropsicología infantil que evalúe y siga esta población hasta finales de la adolescencia. La necesidad es latente por las diferentes alteraciones que no son controladas por el tratamiento temprano. Los estudios informan que las funciones superiores que no son controladas por el tratamiento en la edad preescolar son: las habilidades viso espaciales, el acto motor propositivo y la inhibición conductual (Ramírez y Marchena 2008, 2009).

Los eventos negativos de naturaleza endocrina (Hipotiroidismo congénito, Hiperplasia adrenal congénita e Histidinemia atípica) imponen limitaciones en el desarrollo del sistema nervioso de carácter difuso, aunque con los avances de la edad esas limitaciones van tomando cierta especificidad, esencialmente en las funciones superiores que se apoyan en las regiones frontoparietales. (Álvarez, 2010; Cohen et al., 2005).

\section{Condiciones preacadémicas}

Otro interesante resultado, relacionado con el tercer objetivo de los profesores, fue la capacidad del instrumento para identificar las habilidades preacadémicas. El niño preescolar que presenta dificultades en el dominio ejecutivo de la Luria Inicial presentará limitaciones ejecutivas en la etapa escolar tres años después de aplicado el instrumento (Ramírez y Díaz, 2013). El desarrollo de las funciones viso espaciales e inhibición conductual predicen el estado de la atención y la memoria, sin embargo el desarrollo del acto motor intencional puede predecir el número de perseveraciones en el niño. En conclusiones, las condiciones preacadémicas del niño se obtienen por el estado real de las funciones superiores y las potenciales. Las primeras son aquellas que pueden evaluarse con el instrumento y la segunda se obtiene conociendo la función regulador del habla: habilidad que no solo se evalúa por el test 4 , ella forma parte intrínseca del dominio ejecutivo del instrumento.

Los niños que presentaron alteraciones del neurodesarrollo mostraron alteraciones en las funciones ejecutivas, esencialmente por no tener adquirida la función reguladora del habla. La debilidad limita el salto cualitativo de la edad e impide que el niño verifique los errores en la actividad, siga largas secuencias para ejecutar actividades, organice ejecuciones en ambientes desorganizados con apoyo viso espacial, siga órdenes verbales para ejecutar y aumente las actividades con el apoyo de una guía mental (lenguaje interno). Las limitaciones son importantes para avanzar en la próxima etapa escolar, en especial en las matemáticas (Solovieva, et al., 2013a, Monette et al., 2011) y la lectura (Monette et al., 2011).

La utilidad clínica del instrumento está en la posibilidad de describir - explicar el estado real de las funciones superiores y su potencial: el niño puede ejecutar e imitar movimientos; dibujar, reconocer los colores y hablar (estado real), pero si no organiza esas ejecuciones a través del lenguaje oral regulador (estado potencial) estará sujeto a errores y a no aprender por orientaciones del adulto en la etapa escolar. Otro estudio resalta la importancia del lenguaje oral como condición preacadémica para avanzar en la futura etapa escolar, aunque incluye la memoria como indispensable. (W. Li, Farkas et al., 2013, Cote et al., 2013)

El modelo neuropsicológico de Luria para edades inferiores es un alternativa teórica práctica necesaria para conocer los niveles de organización funcional del sistema nervioso en desarrollo. La organización y regulación de la actividad que van asumiendo los procesos mentales en el niño a través del lenguaje oral es una manera sencilla y válida de evaluar el desarrollo y potenciarlo. Esa regulación se determina por el estado del acto motor y sus niveles de complejidad con el lenguaje oral. Acto motor que adquiere niveles de organización complejos gracias a la maduración cerebral de los lóbulos frontales y parietales en los primeros años, para luego ser los dos lóbulos un sistema funcional a favor de organizar ejecuciones con letras y números. La edad preescolar es crítica para ese tránsito: complejidad en los procesos motores perceptivos acompañado de progresiva jerarquización funcional del lóbulo frontal en las ejecuciones. 
La función reguladora del habla no puede establecerse si existe apraxia, lo cual explica que madura a la par de las funciones que organizan el acto motor, aunque no puede dar un salto cualitativo superior si las ejecuciones motoras de la edad (manos, boca, lengua, pies) no están establecidas. La línea de pensamiento que ofrece Luria para estudiar a los niños es confiable para diagnosticar y rehabilitar las secuelas del sistema nervioso en desarrollo.

El instrumento ofrece un perfil neuropsicológico en la edad preescolar, ahora queda por resolver si es efectivo para predecir el futuro desempeño académico y para seguir terapias de una etapa (preescolar) a otra (escolar). Igualmente, se necesita implementar un proceso de adaptación y normalización del instrumento en el contexto cubano.

\section{Agradecimientos}

Se agradece a los profesores Dionisio Manga y Francisco Ramos por organizar el modelo de Luria en un instrumento, y facilitar a las nuevas generaciones la visión interdisciplinaria de la neuropsicología para edades inferiores.

\section{Conclusiones}

La experiencia de trabajo con el instrumento Luria Inicial permite introducir un instrumento válido para evaluar las condiciones preacadémicas del niño con alteraciones del neurodesarrollo. Organizar una estrategia curricular del niño preescolar exige un diagnóstico de las funciones mentales superiores en desarrollo

1. El modelo neuropsicológico de Luria es una herramienta teórica práctica poderosa para estudiar y seguir el desarrollo de las funciones psicológicas superiores en la edad preescolar

2. El acto motor con intenciones, la inhibición conductual y la organización espacial de las ejecuciones son habilidades superiores diana a cualquier evento que interrumpa el neurodesarrollo: neurobiológico y/o privación educativa.
3. La función reguladora del habla es la habilidad potencial que orienta las funciones psicológicas a niveles superiores de organización en la edad preescolar: niños con/sin alteraciones en el neurodesarrollo pueden presentar atraso en su adquisición.

\section{Referencias bibliográficas}

Adair, L. S., Fall, C. H., Osmond, C., Stein, A. D., Martorell, R., Ramirez, M., COHORTS group (2013). Associations of linear growth and relative weight gain during early life with adult health and human capital in countries of low and middle income: findings from five birth cohort studies. Lancet, 382(9891), 525-534.

Aguilera, E. C., Ostrosky, F. y Camarena, B. (2012). Interacción de Temperamento y MAO-A en pruebas de Inhibición en Preescolares. Acta de Investigación Psicológica, 2(1), 543-554

Alfonso, A., Ramos, M., Alday, C. y Alquinta, A. (2010). Madurez neuropsicológica en preescolares: propiedades psicométricas del test CUMANIN. Terapia psicológica. 28(1), 13-25.

Álvarez, M. (2010). Episodes of over treatment during the first six months in children with Congenital Hypothyroidism and their relationships with sustained attention and inhibitory control at school age. Horm Res Pediatry, 74, 114-120.

Andrews, K., Fang, H., Charak, D., Minich, N. y Taylor, N. G. (2009). Growth Mixture Modeling of Academic Achievement in Children of Varying Birth Weight Risk. Neuropsychology, 23(4), 460-474.

Bausela, E. (2008). Estudio de algunas propiedades psicométricas de la Batería Luria-Inicial en una muestra de escolares mexicanos. Archivos de Neurociencias, 13(4), 232-236.

Bausela, E. y Orozco, C. I. (2009a). Análisis de algunas propiedades psicométricas de la Batería Luria- Inicial en una muestra de escolares potosinos. Boletín de Pediatría, 49, 35-40.

Bausela, E. y Orozco, C. I. (2009b). Estudio piloto de validación de la Batería neuropsicológica Luria-Inicial con el subtest de matrices del WISCIV y el test de matrices progresivas de RAVEN. Boletín Electrónico de Investigación Psicológica 
de la Asociación Oaxaqueña de Psicología, 5(1), 57-66.

Bjuland, K. J., Løhaugen, G. C., Martinussen, M. y Skranes, J. (2012). Cortical thickness and cognition in very-low-birth-weight late teenagers. Early Human Development, 89(6), 371-380. doi: 10.1016/j.earlhumdev.2012.12.003.

Campo, C., Tuesca, R. y Campo, L. (2012). Relación entre el grado de madurez neuropsicológica infantil y el índice de talla y peso en niños de 3 a 7 años escolarizados de estratos socioeconómicos dos y tres de la ciudad de Barranquilla. Salud Uninorte, 28(1), 88-98.

Campo, L. A. (2010). Importancia del desarrollo motor en relación con los procesos evolutivos del lenguaje y la cognición en niños de 3 a 7 años de la ciudad de Barranquilla (Colombia). Salud Uninorte, 26(1), 65-76.

Charach, A., Carson, P., Fox, S., Ali, M. U., Beckett, J. y Lim, C. G. (2013). Interventions for preschool children at high risk for ADHD: a comparative effectiveness review. Pediatrics, 131(5), 1584-1604.

Cherkasova, M., Sulla, E. M., Dalena, K. L., Pondé, M. P. y Hechtman, L. (2013). Developmental course of attention deficit hyperactivity disorder and its predictors. Journal Can Acad Child Adolesc Psychiatry, 22(1), 47-54.

Christensen, A. L. (1987). El diagnóstico neuropsicológico de Luria. Madrid, España: Visor.

Cohen, C. C., Van de Beek, C. y Berembaum, S. A. (2005). Prenatal sex hormone effects on child and adult sex typd behavior methods and findings. Neurosciences and biobehavioral reviews, 29, 353-384.

Côté, S. M., Mongeau, C., Japel, C., Xu, Q., Séguin, J. R. y Tremblay, R. E. (2013). Child care quality and cognitive development: trajectories leading to better preacademic skills. Children Development, 84(2), 752-766. doi: 10.1111/cdev.12007.

Drvaric, L. A., Van Lieshout, R. J. y Schmidt, L. A. (2013). Linking Early Adversity, Emotion Dysregulation, and Psychopathology: The Case of Extremely Low Birth Weight Infants. Child Development Research. http://dx.doi. org/10.1155/2013/203061.

Fernandes, L. V., Goulart, A. L., dos Santos, A. M., Barros, M. C., Guerra, C. C. y Kopelman, B. I.
(2012). Neurodevelopmental assessment of very low birth weight preterm infants at corrected age of 18-24 months by Bayley III scales. Journal Pediatry (Rio J), 88(6), 471-478.

Getahun, D., Jacobsen, S. J., Fassett, M. J., Chen, W., Demissie, K. y Rhoads, G. G. (2013). Recent trends in childhood attention-deficit/hyperactivity disorder. JAMA Pediatr. 167(3), 282-288.

González, M. G. y Ostrosky, F. (2012). Estructura de las Funciones Ejecutivas en la Edad Preescolar. Acta de Investigación Psicológica, 2(1), 509-520.

Goodman, D. M. y Livingston, E. H. (2013). Attention deficit/hyperactivity disorder. Journal of the American Medical Association, 309(17), 1843.

Griffiths, S. T., Gundersen, H., Neto, E., Elgen, I., Markestad, T., Aukland, S. M. y Hugdahl, K. (2013). fMRI: blood oxygen level-dependent activation during a working memory-selective attention task in children born extremely preterm. Pediatr Res., 74(2), 196-205.

Guaita (2012). Funcionamiento neuropsicológico y recuerdo episódico en preescolares chilenos: Un estudio exploratorio descriptivo (Tesis de postgrado). Facultad de Ciencias Sociales. Universidad de Chile. Recuperado de http://www.tesis. uchile.cl/handle/2250/113989

Guerrero, M., Guillén, D. y Sato, L. (2011). Desarrollo Neuropsicológico en niños preescolares con exposición crónica a plomo, residentes en el Callao-Perú. Revista Peruana de Pediatría, 64(2).

Hutchinson, E. A., De Luca, C. R., Doyle, L. W., Roberts, G., Peter, J. y Anderson, P. J. (2013). School-age Outcomes of Extremely Preterm or Extremely Low Birth Weight Children. Pediatrics, 131(4), 780.

June, C. y Diane, D. (2013). Effects of Perinatal Testosterone on Infant Health, Mother-Infant Interactions, and Infant Development. Biol Res Nurs, 16(2), 228-236.

Korkman, M. (1988). An adaptation of Luria s investigation for young children. The Clinical Neuropsychologist, 2, 375-392.

Kwinta, P., Klimek, M., Grudzie区, A., Nitecka, M., Profus, K., Gasi囚ska, M.,... Pietrzyk, J. J. (2012). Intellectual and motor development of extremely low birth weight $(\leq 1000 \mathrm{~g})$ children in the 7 th year of life; a multicenter, cross-sectional study of children born in the Malopolska voivodship 
between 2002 and 2004. Medical Wieku Rozwoj, 16(3), 222-231.

Lee, P. A. y Houk, C. P. (2012). Cognitive and psychosocial development concerns in children born small for gestational age. Pediatr Endocrinol Rev, 10(2), 209-216.

Lezak, M. D. (1984). T Sampling problems and nonparametric solutions in clinical neuropsychological research. Journal Clinical Neuropsychological, 6(1), 101-109.

Li, N., Kang, L. M., Wang, Q., Yu, T., Ma, D. y Luo, R. (2013). Effects of early neurodevelopmental treatment on motor and cognitive development of critically ill premature infants. Sichuan Da Xue Xue Bao Yi Xue Ban, 44(2), 287-290.

Li, W., Farkas, G., Duncan, G. J., Burchinal, M. R. y Vandell, D. L. (2013). Timing of high-quality child care and cognitive, language, and preacademic development. Development Psychology, 49(8), 1440-1451. doi: 10.1037/a0030613

Lohaugen, G. C., Austeng, D., Brubakk, A. M. y Skranes, J. (2013). Working memory training improves cognitive function in VLBW preschoolers. Pediatrics, 131(3), 747-754.

Lorenzo, G. (2011). La motricidad fina en niños preescolares con trastorno por déficit de atención con hiperactividad. Tesis de grado para optar por el título de Psicología. Universidad de Ciencias Médicas de Matanzas. Facultad de Psicología, Matanzas, Cuba.

Lozano, A. y Ostrosky, F. (2012). Efecto del Nivel Socioeconómico en el Control Inhibitorio durante la Edad Preescolar. Acta de Investigación Psicológica, 2(1), 521-531.

Luria, A. R. (1982). El cerebro en acción. La Habana, Cuba: Revolucionaria.

Manga, D. y Ramos, F. (1991). Neuropsicología clínica infantil. Aplicaciones de la teoría de A. R. Luria a niños a través de la batería Luria - DNI. Madrid, España: Visor.

Manga, D. y Ramos, F. (2006). Luria Inicial. Evaluación neuropsicológica en la edad preescolar. Madrid, España: TEA.

Monette, S., Bigras, M. y Guay, M. C. (2011). The role of the executive functions in school achievement at the end of Grade 1. J Exp Child Psychol, 109(2), 158-173.
Pauli-Pott, U., Dalir, S., Mingebach, T., Roller, A. y Becker, K. (2014). Attention deficit/hyperactivity and comorbid symptoms in preschoolers: Differences between subgroups in neuropsychological basic deficits. Child Neuropsychol, 20(2), 230-244.

Portellano, J. A, Mateos, R, Martínez, R., Granados, M. J. y Tapia, A. (2000). Cuestionario de madurez neuropsicológica infantil. CUMANIN. Madrid, España: TEA.

Quintanar, L., Gómez, R., Solovieva, Y., y Bonilla, M. R. (2011). Características neuropsicológicas de niños preescolares con trastorno por déficit de atención con hiperactividad. Revista CES Psicología, 4(2), 16-31.

Ramírez, Y. (2008). Signos neurológicos menores en la edad preescolar. Revista Mexicana de Neurociencias, 9(6), 445-453.

Ramírez, Y. y Díaz, B. (2013). Efecto del bajo peso al nacer sobre el desarrollo cognitivo. Boletín de Pediatría, 53, 13-20.

Ramírez, Y. y González, M. (2008). Estudio neuropsicológico en niños de 6 años con antecedentes de hipoxia perinatal. Archivo de Neurociencias Mex, 13(3).

Ramírez, Y. y Marchena, H. M. (2008). Alteraciones neuropsicológicas en la hiperplasia adrenal congénita. Revista Cubana de Endocrinología, 19(3).

Ramírez, Y. y Marchena, H. M. (2009). Características neuropsicológicas del niño preescolar con Hipotiroidismo Congénito en la Provincia de Cienfuegos. Revista Chilena de Neuropsicología, 4(1), 36-43.

Ramírez, Y. y Novoa, M. (2008). Estudio neuropsicológico en niños de 6 años con antecedentes de hipoxia perinatal. Archivos de Neurociencias (Mex), 13(3), 162-169.

Ramírez, Y., Díaz, B, Ramos, F., Manga, D. (2013). Validez y confiabilidad de la batería Luria Inicial para identificar alteraciones neuropsicológicas en niños cubanos. Revista Cubana Neurología y Neurociencias, 3(1),18-25.

Ramírez, Y., Díaz, M., Hernando, D. y Samoano, R. M. (2011). Batería Neuropsicológica Luria Inicial y procesos atencionales. Revista Chilena de Neuropsicología, 6(1). 
Raz, S., Newman, J. B., Debastos, A. K., Peters, B. N. y Batton, D. G. (2014). Postnatal growth and neuropsychological performance in preterm-birth preschoolers. Neuropsychology, 28(2), 188-201.

Riddle, M. A., Yershova, K., Lazzaretto, D., Paykina, N., Yenokyan, G., Greenhill, L.,... Posner, K. (2013). The Preschool Attention-Deficit/Hyperactivity Disorder Treatment Study (PATS) 6-year follow-up. Journal Am Acad Child Adolesc Psychiatry, 52(3), 264-278.

Ritter, B. C., Perrig, W., Steinlin, M. y Everts, R. (2013). Cognitive and behavioral aspects of executive functions in children born very preterm. Child Neuropsychology, 20(2), 129-144.

Rodríguez, J. P. (2008). Algoritmo para la atención temprana de infantes con factores de riesgo de afectaciones del neurodesarrollo. Tesis maestría «Atención integral al niño». Universidad de Ciencias Médicas de Matanzas.

Sandoval, C. y Ostrosky, F. (2012). Efectos del polimorfismo Val108/158Met del gen dopaminérgico Catecol-O-metil transferasa COMT en las funciones ejecutivas de preescolares. Revista Chilena. Neuropsicología, 7(3), 115-121.

Schmiedeler, S. y Schneider, W. (2013). Attentiondeficit hyperactivity disorder (ADHD) in the early years: Diagnostic issues and educational relevance. Clinical Child Psychology and Psychiatry. Recuperado de http://ccp.sagepub.com/content/early/2013/06/11/1359104513489979.long

Serrano-Troncoso E., Guidi M. y Alda-Díez J. A. (2013). Is psychological treatment efficacious for attention deficit hyperactivity disorder (ADHD)? Review of non-pharmacological treat- ments in children and adolescents with ADHD. Actas Esp Psiquiatr, 41(1), 44-51.

Solovieva, Y, Lázaro, E. y Quintanar, L. (2008). Mecanismos de los lóbulos frontales en niños preescolares con déficit de atención y niños normales. Acta Neurológica Colombiana, 24, 64-75.

Solovieva, Y. y Quintanar, L. (2013). Evaluación del desarrollo simbólico en niños preescolares mexicanos. Cultura y Educación, 25(2), 167-182.

Solovieva, Y., Emelia, E. y Quintanar, L. (2013). Evaluación de las habilidades matemáticas previas en niños preescolares urbanos y rurales. Cultura y Educación, 25(2), 199-212.

Solovieva, Y., Loredo, D., Quintanar, L. y Lázaro, E. (2012). Caracterización neuropsicológica de una población infantil urbana a través de la Evaluación Neuropsicológica Infantil Puebla-Sevilla. Pensamiento Psicológico, 11(1), 83-98.

Somoano, R. M., Ramírez, Y., Díaz, M. y Falcón, F. (2012). Histidinemia atípica y desarrollo cognitivo. Revista Cubana de Endocrinología, 23(2), 157-165.

Takayanagi, T., Egashira, M., Yamaguchi, T., Murata, N., Yokota, G., Matsuo, K.,... Mizukami, T. (2013). Cognitive outcome of very low birth weight infants at 6 years of age. Pediatr Int, 55(5), 594-598.

Walhovd, K. B., Fjell, A. M., Brown, T. T., Kuperman, J. M., Chung. Y., Hagler, D. J. Jr,... Dale, A. M. (2012). Long-term influence of normal variation in neonatal characteristics on human brain development. Proc Natl Acad Sci, 109(49), 20089-20094. 
